\title{
Selection of enterprise resource planning (ERP) systems
}

\author{
D. das Neves, D. Fenn \& P. Sulcas* \\ Graduate School of Business, University of Cape Town \\ Private Bag, Rondebosch 7701, Republic of South Africa \\ psulcas@gsb.uct.ac.za
}

Received November 2003

In order to determine the process organisations go through in selecting an Enterprise Resource Planning (ERP) system, a field study was undertaken on eleven cases. Based on the findings a best practice selection process is proposed.

\begin{abstract}
A benchmark selection criteria checklist was drawn up as part of this investigation and each of the criteria listed should be considered prior to final selection.
\end{abstract}

Other issues discussed in this article include the original motivation and justification for ERP purchase, change management, customisation of source code and the roles of the selection committee, consultants and vendors.

*To whom all correspondence should be addressed.

The names of the organisations used in this investigation have not been disclosed for reasons of confidentiality.

\section{Introduction}

Enterprise Resource Planning (ERP) is an information technology software system, integrating business processes to improve speed and accessibility of information flows inside an organisation. However, the acquisition of ERP software is not without its challenges as it is a highexpenditure activity and if a poor choice is made, it can adversely affect the organisation as a whole, even jeopardising its very existence. This highlights the need for performing adequate levels of research into making the correct choice of software and preparing the organisation for its introduction.

The literature survey revealed the paucity of relevant South African literature in the subject, which could result in senior mangers being held hostage to the experience and opinions of their IT staff and outside consultants. This investigation aids management by exploring possible shortcomings into current selection procedures and offers relevant experience that should be considered when selecting an ERP system.

The authors studied the process that a number of organisations underwent in the selection of an ERP system and commented on the experience gained. The good news is that in most of the recent cases examined, the end result of the selection process was considered to be a success. The speed of the selection process has increased in recent years and fewer omissions of important criteria made. The checklist drawn up as part of this research draws on this experience and will aid senior managers in their own selection decisions. A warning is sounded around the issue of change management as this area is often not properly thought out prior to implementation.

\section{Literature review}

Tanner (2002) argues that correct ERP selection is vital to minimise financial risk and uncertainties about the software and its compatibility with the organisations' business structure. Slater (1999a: 4-5) concurs with this view writing that: 'choosing the wrong one [ERP package] can be a costly disaster' and recommends using a selection checklist.

Verville and Harlingten (2002: 207) add that the acquisition process for ERP systems is important as it allows examination of all the dimensions and implications (benefits, risks, challenges, costs, etc.) prior to the commitment of formidable amounts of money, time and resources.

A literature review showed that some organisations perform inadequate research, both in terms of time and spending, before choosing an ERP system. The main problem areas were:

(i) Inadequate financial research

ERP implementation often results in intangible benefits that are difficult to evaluate rigorously using financial tools such as Return on Investment (ROI) (Scott, 1997). Faced with this difficulty, many companies may opt out of this evaluation process altogether (Thornton, 1996). This implies that an ERP system with higher upfront costs or less obvious future benefits may be rejected for an inferior product (Slater, 1999b).

(ii) Functionality constraints

Slater (1999a: 6) wrote, 'Every ERP selection process eventually gets down to the search for individual features and functions', which, if not identified, could '....transform 
an otherwise great fit into a complete mismatch'. He gives examples of organisations buying an ERP system only to find a vital element missing: for example, the lack of multicurrency capability in a large global concern, resulting in it scrapping the system. Some organisations accept that no off-the-shelf product will exactly fit their purpose and rely on customising capability to complete the replacement of the legacy system (Davenport, 1998).

\section{(iii) Time constraints}

Time has become a major factor in tempting organisations to cut corners. For example, in the run-up to the millennium, organisations rushed through the implementation process to beat that particular Y2K deadline (Towns, 2000). Also, some companies rush their IT strategies to maintain or gain competitive advantage without performing sufficient preparatory work (Fang Yih, 2001).

\section{(iv) Skill constraints}

Management in many organisations can mistakenly assume that the necessary skills to choose an ERP system already exist within their organisation (Donovan, 2000). This fallacy can lead to the eventual overuse of outside consultants who may not be totally objective in the choice of ERP systems.

\section{(v) Complacency}

ERP has been around for a long while and there have been many case studies on the implementation of ERP systems (Verville \& Harlingten, 2002; Laudon \& Laudon, 2002; Slater, 1999a). This may lead some organisations to believe that most if not all the lessons of past mistakes have been learnt. However, mergers and acquisitions have introduced many new market players in the last few years. There are currently twenty seven major ERP vendors and the number is rising (Accenture, 2001). The latest push has been towards integrating ERP with the Internet. This has lead to a plethora of new versions and niche modules (Fox, 2000). These changes strongly suggest that any organisation wishing to purchase or upgrade their ERP system should still perform basic selection research to avoid dissatisfaction.

\section{(vi) Selection committees}

Collins (2002b: 1) warns that 'For larger companies, it is important to establish a steering committee to oversee the evaluation and selection process...5 to 7 people - no more. This committee does not actually do any work - they merely 'steer'. The steering committee should prepare the budget and make the final selection'. Within the committee, Donovan (2000: 3) stresses that an executive-level ERP champion is vital to keep top management in the decision loop.

Collins (2002b) also stated that it is very popular for IT staff to recommend products because they think the experience will look good on their résumé. This suggests that the original motivation and motivator be questioned to ensure that the proposed selection is in the best interest of the organisation.

\section{(vii) Scalability}

The selection procedure should be dependant on the scale of the intended ERP installation. The complexity of the impact of an ERP purchase to an organisation ranges from the upgrade of a single module to that of an entire ERP system within a Business Process Reengineering (BPR) initiative. It also depends on how embedded the system will be with regards to corporate culture.

Intuitively, if an organisation merely upgraded their version of software, then one can suppose that if they were satisfied with their current software and service, they would simplify the selection procedure by retaining the same vendor as before without having to alter the company culture.

In parallel to the complexity of re-engineering planned, the size of the organisation affects the selection procedure. Benroider and Koch (2000: 1028) state that smaller organisations would normally require simpler systems than larger organisations.

This information has been summarised in a matrix (Figure 1). The figures used are relative. Synthesising the literature survey into a matrix form, demonstrates why a higher alphanumeric figure should command a more comprehensive selection procedure. The highlighted blocks indicate the position of the organisations investigated in this study. To improve comparisons, the organisations were targeted around the larger and more complex selections.

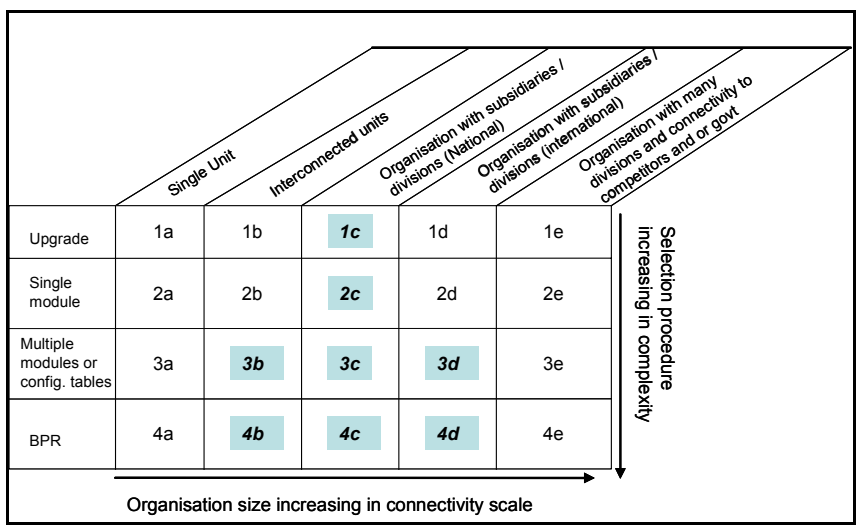

Figure 1: Matrix of size and complexity of selection procedure with position of organisation examined in this investigation highlighted

\section{(viii) Role of consultants}

Collins (2002b: 1-2) writes: 'Depending on the size of your company, the scope of the solution needed, your knowledge, and the available time of you and your staff, hiring an independent consultant can be a good move. It allows you to capitalize on the consultant's expertise and gives you someone to blame if the system eventually does not work out...You might be better served to narrow down the selection first on your own, and then bring in consultants for each product'.

This rather negative view of consultants as being of only limited use and not providing value for money is countered by Damgaard (2002: 4) who writes 'Working with an external consultant who is skilled in mapping business 
processes ensures that you accurately identify all core needs and find a solution that fits them'.

Using a checklist, that highlights all the necessary criteria to be considered before selection, may allow the consultants to be more involved with practical selection and implementation decisions, rather than in passive support roles.

\section{(ix) Vendor influence}

Collins (2002b) recommends attending meaningful demonstrations at live sites from more than one vendor. Damgaard (2002) concurs and suggests asking direct questions. Both suggest that organisations must not believe everything vendors tell them. The role of vendors has changed in recent years, moving closer to that of consultants.

\section{Methodology}

An international literature review was performed and a benchmark of all the pertinent aspects that must be considered prior to selection was made. Using the benchmark as a foundation, a comprehensive questionnaire was drawn up to test if each criterion was considered by the test organisations (see Table 1). Eight organisations covering eleven separate ERP selection and implementations were examined in face-to-face interviews using the questionnaire. Additionally, the three largest local vendors (SAP, Oracle, J.D. Edwards), two independent consultants, and other IT experts were interviewed to test the veracity of the questionnaire, benchmark checklist and provide background information. The results were then compared to the benchmark selection criteria checklist. Subsequent to the analysis, conclusions and recommendations were made.

\section{Findings and analysis of interviews}

Table 2 summarises the industry in which each organisation in the sample operates and includes the ERP software selected.

\section{Table 1: Benchmark selection criteria checklist}

\begin{tabular}{ll}
\hline $\mathbf{1 . 0}$ & Vendor (and stakeholders) evaluation \\
\hline 1.1 & Number of installations performed by vendor locally \\
\hline 1.2 & Market rating, market share, reputation, strength and sustainability of vendors \\
\hline 1.3 & Generic demonstration by vendor of previous ERP implementations \\
\hline 1.4 & Local representation and support (software and consultant) \\
\hline 1.5 & Total cost of ownership of ERP system (software, hardware, training etc.) \\
\hline 1.7 & Aveed and ease of ERP implementation from conception to completion of project \\
\hline 1.8 & Independent consultants (specialisation and bias) \\
\hline 1.9 & Composition of selection committee including Champion and Motivator \\
\hline $\mathbf{2 . 0}$ & Functionality of proposed ERP system \\
\hline 2.1 & Proportion of functional requirements to run business \\
\hline 2.2 & Fit to present and/or desired organisation culture (consultation all stakeholders) \\
\hline 2.3 & Fit to business strategy and ability to gain competitive advantage \\
\hline 2.4 & Range of modules that can be added as the business requirements change \\
\hline 2.5 & Increased transparency and information flows with respect to targets and goals \\
\hline $\mathbf{3 . 0}$ & Technical aspects of proposed ERP system \\
\hline 3.1 & Technical requirements and degree of redundancy of legacy system \\
\hline 3.2 & Adaptability and flexibility with respect to any remaining legacy system \\
\hline 3.3 & Robustness of software and ease/cost of maintenance \\
\hline 3.4 & Customisation potential of system to meet requirements, present and future \\
\hline 3.5 & User friendliness of system \\
\hline 3.6 & Future Upgrade potential of system \\
\hline & \\
\hline 1.6
\end{tabular}


Table 2: Industry and ERP selected by case

\begin{tabular}{l|l|l}
\hline \multicolumn{1}{c|}{ Case } & \multicolumn{1}{c|}{ Industry } & ERP selected \\
\hline $\mathrm{A}$ & Banking & J.D. Edwards \\
\hline $\mathrm{B}$ & Medical supplier & Great Plains \\
\hline $\mathrm{C}(1)$ & Insurance & Oracle \\
\hline $\mathrm{D}(2)$ & Insurance & SAP HR \\
\hline $\mathrm{E} \mathrm{(3)}$ & Insurance & SAP R/3 \\
\hline $\mathrm{F} \mathrm{(1)}$ & Petroleum and Oil & SAP R/2 \\
\hline $\mathrm{G} \mathrm{(2)}$ & Petroleum and Oil & SAP R/3 \\
\hline $\mathrm{H}$ & Food and allied services sector & J.D. Edwards \\
\hline $\mathrm{I}$ & Mining & SAP R/3 \\
\hline $\mathrm{J}$ & Plastics & J.D. Edwards \\
\hline $\mathrm{K}$ & Insurance & SAP HR \\
\hline
\end{tabular}

It could be assumed that if organisations considered all the items contained in the benchmark checklist, then they would have performed adequate research. A statistical approach to judging success of each case could not be made due to the small sample population. Therefore, a subjective conclusion was made on a case-by-case basis, taking into account the number and relative importance of omissions by each organisation during their selection. In practice every organisation that considered more than $80 \%$ of the selection criteria, was deemed to have performed adequate selection research.

\section{Summary}

Nine of the eleven cases examined were judged to have been successful, as they appeared to have considered most or all the important selection criteria given in the benchmark selection criteria checklist, Table 1 . In summary, the two failed cases ( $D$ and F) were chiefly due to the lack of change management resulting in employee resistance to change. Other cases also indicated that better change management could have improved the implementation process. Most performed some financial analysis, but as the purchase was considered to be strategic, the results were often used simply to leverage costs down rather than to terminate the project. Seven of the cases reported that the main reason for selecting an ERP system was because it fitted the organisation's strategic culture.

\section{- $\quad$ Change management}

Case $F$ failed due to inadequate provision for change management by the parent organisation that drove the selection. In the second selection by the same organisation, some 10 years later, the selection was more in-house-driven, resulting in a much faster and cost effective implementation. Case I emphasised that the change management aspect of the selection was run as a business project rather than an IT project. This concurs with Laudon and Laudon's (2002) view that, Information Systems are too important to be left to a small technical group in an organisation. Even so, the interviewee remarked that 'there was no training strategy and cost predictions were not good'. He also mentioned that in retrospect, the organisation culture should have been part of the selection criteria, as SAP is not flexible and is different to the culture of the organisation. The impression gained during the interviews suggested that the single most important area of selection research that could have been improved was around the question of change management.

The other failed case (D) was very deficient with respect to change management issues such as training and cost. The result was that the implementation stalled due to deadlines not being met and the budget being exhausted.

- Best of breed (amalgamation of ERP modules from various vendors)

Only case K adopted a best of breed approach using SAP for the core and Oracle for its better Internet link and also a middleware translator. This complicated system was deemed successful due to the level of pre-planning in the selection phase.

\section{- Consultants}

Consultants were generally kept at arms length due to the wealth of experience in ERP systems within the organisations. The vendors' influence was also kept to a minimum by inviting ERP business partners to quote. In these cases, the vendors were often used in an oversight role.

- Selection committee

The length of the selection was mostly less than five months, explaining the relative stability of the committees. The experience of the committees was often good enough to exclude consultants from most of the decision making process. The speed of the process was ascribed to the high degree of technical and functional requirement being met by all the main systems, allowing the shortlist to be quickly pared to just one 'Front Runner'. Unless a reason was found for its termination, the front runner was the system usually adopted. Predictably, the three largest vendors were the most popular. See Figure 2 below. In addition to the ERP systems, ten non-ERP systems were considered at various times, but discounted. 


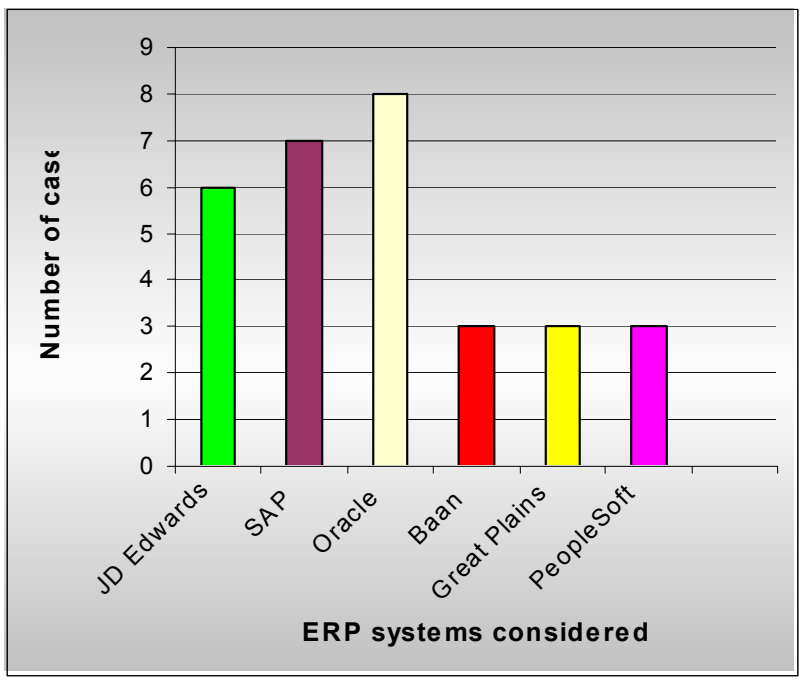

Figure 2: ERP systems considered by type

\section{- Vendor evaluation}

Almost all of the cases asked for request for proposals and considered the size and reputation of the vendor and the availability of skilled support. Additionally, four cases also used the following in their selection: Test pack; Presentations; Cost of ownership; Track record of previous implementations.

- Functionality and customisation

All the organisations that had some missing functionality customised the ERP variable tables, but not the source code. Vendors were generally willing to customise the system, but with eight of the cases, the clients were warned by the vendors that customisation could lead to problems especially when upgrading systems. Request for customisation (development requests) in SAP normally come out as standard offerings in new releases. Oracle appeared to be more willing to customise but this has led to reported problems in their implementations

A summary section was included in the questionnaire encompassing all the criteria in the benchmark checklist. The responses were enlightening and are detailed below.

\section{- A. Vendor selection}

As can be seen in table 3 almost every case scored well in this section. The three cases who reported that the ERP did not fit their culture were in the process of BPR programmes and so a change in culture was one of the accepted outcomes. Cases D and G both stated that the total cost of ownership (TCO) was irrelevant as the purchase was a strategic necessity. Case $\mathrm{F}$ had prior experience of installations and so expressed an opinion that the eventual cost was acceptable, but case D had not completed their installation as their budget had run out, with no new funds forthcoming. This indicated that as such an important element of the selection process was flawed; this particular case had performed inadequate research prior to implementation. Case $\mathrm{F}$ is unusual as selection research performed was carried out by the parent organisation. Their selection was difficult, which reflects the lack of planning and change management research required to implement a system. It also resulted in the authors declaring the selection a failure.

From the results of this section and the information gleaned from the previous questions, it can be concluded that all the organisations completed at least $80 \%$ of the checklist (Table $1)$. However, it is also concluded that case $D$ and case $F$ performed inadequate research prior to vendor selection.

- $\quad$ B. Functionality of proposed ERP system

The results are displayed in table 4 below. Predictably, most of the cases considered the functionality of their requirement versus that offered by the various ERP packages. Cases C, D and $\mathrm{E}$ purchased an ERP system as part of their BPR programme and so decided that the organisation's culture could be adapted to fit the ERP system. Therefore the fit to organisation culture was considered irrelevant. All the items on the checklist (Table 1) were considered by over $80 \%$ of the cases (counting irrelevance as a consideration), thus it is concluded that functionality is generally well researched by organisations prior to selection. It is noticeable that case D failed to consider two items, which again suggests a paucity of research. Case $\mathrm{H}$ also failed to consider two items, however go-live was still to take place and no conclusions could be made.

\section{- C. Technical aspects of the system}

This section (see Table 5 below) indicates that most items of a technical nature were taken into consideration. The technical requirements for case $\mathrm{K}$ were not considered because the respondents claimed that with their previous ERP experiences, they were confident that all three of the systems they considered would satisfy all the necessary technical functions. Thus in that case, the requirement was considered, but not evaluated. Case D found during implementation, that they should have spent more time on the customisation aspect, as this was the area where they experienced most problems. Case G's selection was easy as it was another upgrade of an existing system. Therefore the system was well known by users and upgrades an accepted part of the IT management. All five respondents that ranked this section stated that robustness of the system was the most important criteria.

Overall, it can be concluded from this section that all aspects were considered at least implicitly, except for the customisation potential of case $\mathrm{D}$, whose inadequate research played a part in the subsequent implementation problems.

In summary of this section, the change management aspect of the selection process was given as an imperative by most of the respondents and that the fit of the new paradigm should meet the overall organisation strategic objectives. The degree of functionality offered by all the major systems reduces the incidence of customisation and allows the selection process to be 'evolutionary' and goal based, not criteria driven. As would be expected, technical aspects were carefully evaluated. 
Table 3: Summary of vendor selection criteria considered ${ }^{1}$

\begin{tabular}{l|l|l|l|l|l|l|l|l|l|l|r|r|r}
\hline & A & B & C & D & E & F & G & H & I & J & K & Total & \% \\
\hline A. Vendor evaluation & & & & & & & & & & & & & \\
\hline Number of installations & 1 & 1 & 1 & 1 & 1 & & 1 & 1 & 1 & 1 & 1 & $\mathbf{1 0}$ & $\mathbf{1 0 0}$ \\
\hline Market rating etc. & 1 & 1 & 1 & 1 & 1 & & 1 & 1 & 1 & 1 & 1 & $\mathbf{1 0}$ & $\mathbf{1 0 0}$ \\
\hline Demonstration & 1 & 1 & 1 & 1 & 1 & & 1 & 1 & 1 & 1 & 1 & $\mathbf{1 0}$ & $\mathbf{1 0 0}$ \\
\hline Local representation & 1 & 1 & 1 & 1 & 1 & & 1 & 1 & 1 & 1 & 1 & $\mathbf{1 0}$ & $\mathbf{1 0 0}$ \\
\hline Total cost of ownership & 1 & 1 & 1 & & 1 & & & 1 & 1 & 1 & 1 & $\mathbf{8}$ & $\mathbf{8 0}$ \\
\hline Speed \& ease & 1 & 1 & 1 & 1 & 1 & & 1 & 1 & 1 & & 1 & $\mathbf{9}$ & $\mathbf{9 0}$ \\
\hline Availability of live site visits & 1 & 1 & 1 & 1 & 1 & & 1 & & 1 & 1 & 1 & $\mathbf{9}$ & $\mathbf{9 0}$ \\
\hline
\end{tabular}

Table 4: Summary of functionality selection criteria considered

\begin{tabular}{l|l|l|l|l|l|l|l|l|r|r|r|r|r}
\hline & A & B & C & D & E & F & G & H & I & J & K & Total & \% \\
\hline B. Functionality of proposed ERP system & & & & & & & & & & & & & \\
\hline Functional requirements & 1 & 1 & 1 & 1 & 1 & 1 & 1 & 1 & 1 & 1 & 1 & $\mathbf{1 1}$ & $\mathbf{1 0 0}$ \\
\hline Fit to organisation culture & 1 & 1 & & & & 1 & 1 & 1 & & 1 & 1 & 7 & $\mathbf{6 4}$ \\
\hline Fit to business strategy & & 1 & 1 & 1 & 1 & 1 & 1 & & 1 & 1 & 1 & $\mathbf{9}$ & $\mathbf{8 2}$ \\
\hline Range of modules that can be added & 1 & 1 & 1 & & 1 & 1 & 1 & & 1 & 1 & 1 & $\mathbf{9}$ & $\mathbf{8 2}$ \\
\hline Incr. transparency \& info. flows & 1 & 1 & 1 & 1 & 1 & 1 & 1 & 1 & 1 & 1 & 1 & $\mathbf{1 1}$ & $\mathbf{1 0 0}$ \\
\hline
\end{tabular}

Table 5: Summary of technical aspects selection criteria considered ${ }^{2}$

\begin{tabular}{l|r|r|r|r|r|r|r|r|r|r|r|r|r}
\hline & A & B & C & D & E & F & G & H & I & J & K & Total & \% \\
\hline C. Technical aspects of proposed ERP system & & & & & & & & & & & & & \\
\hline Technical requirements & 1 & 1 & 1 & 1 & 1 & & 1 & 1 & 1 & 1 & & $\mathbf{9}$ & $\mathbf{9 0}$ \\
\hline Adaptability \& flexibility & 1 & 1 & 1 & 1 & 1 & & 1 & 1 & 1 & 1 & 1 & $\mathbf{1 0}$ & $\mathbf{1 0 0}$ \\
\hline Robustness and ease/cost & 1 & 1 & 1 & 1 & 1 & & 1 & 1 & 1 & 1 & 1 & $\mathbf{1 0}$ & $\mathbf{1 0 0}$ \\
\hline Customisation potential & 1 & 1 & 1 & & 1 & & 1 & 1 & 1 & 1 & 1 & $\mathbf{8}$ & $\mathbf{8 0}$ \\
\hline User friendliness of system & 1 & 1 & 1 & 1 & 1 & & & 1 & 1 & 1 & 1 & $\mathbf{9}$ & $\mathbf{9 0}$ \\
\hline Future upgrade potential of system & 1 & 1 & 1 & 1 & 1 & & & & 1 & 1 & 1 & $\mathbf{8}$ & $\mathbf{8 0}$ \\
\hline
\end{tabular}

\section{Overall findings}

These findings answer the questions and issues raised in the literature review, points i - ix.

\section{Inadequate financial research}

All respondents stressed, in responding to 'total cost of ownership' (refer Table 3), that the need to implement an ERP system was a 'need to have', based on strategic grounds. In most cases, cost benefits were not considered an important criterion and thus the expected inadequate financial research is probably not an important issue in the organisations tested. It is possible that manufacturing organisations, who claim cost benefits as a reason for choosing an ERP system, may indeed perform inadequate financial evaluations - personal communication with a consultant from Deloitte \& Touche.
However, in response Matthias Mueller, Regional Manager from SAPSA disagreed, stating that: 'Today's selection processes are more focused on ROI and total cost of ownership (TCO) than in earlier days'. (Mueller, E-mail, 20/11/2002)

\section{Functionality constraints}

Only one organisation admitted having less than $80 \%$ functionality and none claimed to have been stymied by a terminal functionality gap. Issues surrounding customisation and some minor add-on programmes (for example, report writers) were noted. In general, customising the source code appears to be a frequent cause of problems, especially during software upgrades. It is concluded that choosing a system with most or all the required functionality appears to be the best (and most common) option and waiting for an upgrade to address the functional gap is the second best option.

\footnotetext{
${ }^{1}$ Note that the respondent for case $\mathrm{F}$ was unable to fill out the section due to the age of the selection (1991).

${ }^{2}$ Note that the respondent for case $\mathrm{F}$ was unable to fill out the section due to the age of the selection (1991).
} 
In South Africa, Mueller responded that: 'In SAP terms, customisation refers to the setting of parameters to get the software to react in certain ways. On the other hand, modifications (source code changes) are strongly discouraged by SAP, as these can impact the customer's future upgrade path. Therefore we urge our customers not to tamper with the source code. They can instead rather provide us with a specification (Development request) and we will endeavour to make this part of our standard offering with the next release of the software'. (Mueller, E-mail, 20/11/2002)

\section{Time constraints}

Time constraints appear to sharpen the selection process and actually allows for a more stable selection committee, due to less chance of members leaving. No cases of 'cutting corners' were found, although change management issues could have benefited from more time. Two cases cited Y2K as reasons for purchasing an ERP system, but both were largely successful.

\section{Skill constraints}

Most of the larger organisations claimed they had adequate internal skills availability. The perceived lack of technical support from all the vendors, except SAPSA, was cited as an issue and this constraint highlights the need to avoid customising the ERP code. The lack of technical support in the small Western Cape market may not exist in larger markets. This facet of the investigation may not be representative of the larger South African population.

\section{Complacency}

No evidence was directly found indicating that complacency in the selection process had led to inferior products being purchased. The general feeling amongst the respondents and experts was that all three major ERP systems can perform adequately. There is strong evidence that the better implementations arose from a result of pre-planning of the human aspects of the implementation, especially change management. Cases D and F confirm this conclusion.

\section{Selection committees}

In general, the speed of ERP implementations globally has increased in the last five years and the reduction in selection time has led to a more stable committee. This also appears to be the case in South Africa (CIO - case G, personal communication, October 2002). This research indicates that the total time from inception to completion of the ERP installation averaged less than twelve months except for case D, which stalled. All the respondents claimed that their selection committees were essentially stable, compact and composed of senior IT and non-IT personnel. This stability allows accountability and ordered selection procedures to be followed, thus lessening the risk of important criterion being omitted by oversight.

\section{Scalability}

Since the investigation focused on ERP selections of similar size and scope, no firm conclusions could be made of inadequate selection due to scale.

\section{Role of consultants}

Consultation with all relevant stakeholders is a major consideration in successful selection procedures. However, the use of independent consultants did not appear to add materially to the success rate of selections and this concurs with the literature review. The use of consultants as business partners appears to be on the increase, bringing outside experts closer to the 'coal face'.

\section{Vendor influence}

It is in the vendors' best interest to provide adequate information, data and resources to ensure a smooth implementation. Their influence on the purchase of ERP systems is minimal as the selection is often made prior to the vendors being approached. As stated by Mueller, (Personal contact by e-mail on 20/11/2002), the vendors are increasingly being used to oversee the selection and implementation process as it is in their interests to ensure a smooth process and enhancement of their reputation.

SAPSA reports that a consortium approach is not uncommon and that SAP offers its Quality Assurance services to all implementations [an overseeing role]. It uses consultants and the purchaser in cooperative strategic alliances. SAPSA differentiates between three types of strategic alliances: Complimentary Software partners, Technology partners and Service partners (for example, Application Service Providers); these all form part of the SAP Eco-system. (Mueller, E-mail, 20/11/2002)

\section{Conclusion}

The cost, complexity and potential complications of implementing an ERP imply that serious consideration must be given to the selection process as well as between competing products.

Based on a comprehensive literature survey, little evidence was found as to the process organisations go through in making this selection. But what did emerge from analysing and synthesising available literature was a benchmark of key variables that could be used - see Table 1 . In order to validate these research findings, a questionnaire was developed and used as the basis for semi-structured interviews in eight sites that installed eleven separate ERP systems.

The findings clearly indicate that there is a consistent approach to successful selection processes in that the items in Table 1 are considered prior to deciding which ERP vendor to select. This reflects the inclusion of lessons learnt from historic selection processes. It is concluded that any organisation considering purchasing an ERP system should consider the same variables. 
It should be noticed that for various reasons the fieldwork was conducted on a relatively small number of organisations, all of which are in the Western Cape. It is felt that this does not minimise or invalidate the findings of this study. But it does create an opportunity to extend research on the topic into a wider geographical (national and international) market place.

\section{Recommendations}

1. This research and the benchmark selection criteria checklist should be made freely available to all organisations, so they can check if all the major items to be considered during selection have been covered. This will be particularly useful in cases where the vendors and outside consultants are not used or have little influence on the recommendations of the selection committee.

2. In addition to the benchmark checklist, all the issues surrounding change management should be examined as part of the selection process.

3. Customisation of source code should be undertaken only after the purchasing organisation has been made fully aware of the potential problems that may be encountered when upgrading.

\section{References}

Accenture. 2001. 'ERP market 2001'. Reference DRT2344.PPT. Unpublished presentation.

Benroider, E. \& Koch, S. 2000. 'Differences in characteristics of the ERP system selection process between small or medium and large organizations'. Proceedings. of the Sixth Americas Conference on Information Systems (AMCIS 2000): 1022-1028.

CIO - case G. 2002. Personal communication on 22/10/2002.

Collins, C. 2002a. 'The fast track to the right solution'. [online]

URL: http://www navision.com/hq/view.asp?documentID=819.

Collins, J.C. 2002b. '23 Steps for choosing the right ERP accounting software package'. [online] URL: http://www.accountingsoftwarenews.com/find/find $20 \mathrm{htm}$.

Damgaard, P. 2002. 'A step-by-step Guide to choosing the right business solution'. [online] URL: http://www navision.com/hq/view.asp?documentID=1036.

Davenport, T.H. 1998. 'Putting the enterprise into the enterprise system’. Harvard Business Review, 76(4):121133.

Davenport, T.H. 2001. Forward enterprise integration, New York: John Wiley.
Donovan, R.M. 2000. 'Successful ERP implementation the first time'. [online] URL: http://www rmdonovan.com/pdf/perfor8.pdf.

Fang Yih, T. 2001. 'Old business rules, rule'. [online] URL: http://www.cio-

asia.com/pcio.nsf/unidlookup/FF0D9EF2F86D55A348256B 4A0041A488? OpenDocument

Fox, J. 2000. 'Lumbering toward B2B'. [online] URL: http://www.cnn.com/SPECIALS/2000/e.europe/stories/b2b/

Laudon, K.C. \& Laudon, J.P. 2002. Management information systems: Managing the digital firm. $7^{\text {th }}$ Edition. New Jersey: Prentice-Hall, Inc.

Mueller, M. 2002. Personal contact by e-mail on 20/11/2002.

Scott, J.M. 1997. 'An investigation into the justification of major IT expenditure in selected companies in the Western Cape'. Unpublished MBA research report, GSB, University of Cape Town.

Slater, D. 1998. 'The hidden costs of enterprise software'. [online] URL: http://www.cio.com/archive/enterprise/011598 erp.html

Slater, D. 1999a. 'An ERP package for you... and you... and you....and even you'. [online] URL: http://www.cio.com/archive/021599/erp html

Slater, D. 1999b. 'The only things you shouldn't worry about'. [online] URL: http://www.cio.com/archive/021599/erp html

Taitz, L. 1998. 'SAP R/3: Changing the role of the IT department'. Unpublished MBA research report, GSB, University of Cape Town.

Tanner, A. 2002. 'Enterprise resource planning: The risks of ERP'. [online] URL: http://www.du.edu/ atanner/index htm.

Thornton, G. 1996. 'ROI who?'. [online] URL: http://www.computerworld.com/news/1996/story/0,11280,1 $\underline{6301,00 \mathrm{html}}$

Towns, S. 2000. 'Greased lightning'. [online] URL: http://www.govtech net/magazine/gt/2000/aug/erp/greased.p $\underline{\mathrm{html}}$

Verville, J. \& Harlingten, A. 2002. 'An investigation of the decision process for selecting an ERP software: The case of ESC', Management Decision, 40(3): 206-216.

Unknown. 2002. 'ERP Business Partners (EBP)'. [online] URL: $\underline{\text { http://www.vansomeren.com/erp/providers/index.htm. }}$ 